\title{
Understanding multilayers from a geometrical viewpoint
}

\author{
T. Yonte, J. J. Monzón, L. L. Sánchez-Soto \\ Departamento de Óptica, Facultad de Ciencias Físicas, Universidad Complutense, 28040 Madrid, Spain \\ J. F. Cariñena \\ Departamento de Física Teórica, Facultad de Ciencias, \\ Universidad de Zaragoza, 50009 Zaragoza, Spain \\ C. López-Lacasta \\ Departamento de Matemática Aplicada, CPS, Universidad de Zaragoza, 50015 Zaragoza, Spain.
}

(Dated: November 14, 2018)

\begin{abstract}
We reelaborate on the basic properties of lossless multilayers. We show that the transfer matrices for these multilayers have essentially the same algebraic properties as the Lorentz group $\mathrm{SO}(2,1)$ in a $(2+1)$-dimensional spacetime, as well as the group $\mathrm{SL}(2, \mathbb{R})$ underlying the structure of the $A B C D$ law in geometrical optics. By resorting to the Iwasawa decomposition, we represent the action of any multilayer as the product of three matrices of simple interpretation. This group-theoretical structure allows us to introduce bilinear transformations in the complex plane. The concept of multilayer transfer function naturally emerges and its corresponding properties in the unit disc are studied. We show that the Iwasawa decomposition reflects at this geometrical level in three simple actions that can be considered the basic pieces for a deeper undestanding of the multilayer behavior. We use the method to analyze in detail a simple practical example.
\end{abstract}

PACS numbers: 120.5700 Reflection, 120.7000 Transmission, 230.4170 Multilayers, 000.3860 Mathematical methods in physics

\section{INTRODUCTION}

Layered media are important for many applications in modern optics. To fully capitalize these media one must have a clear picture of all the mechanisms involved in the propagation of the optical waves in them. In spite of this fact, multilayer optics is usually approached from a practical view, in relation to optical filters and the like. In this spirit, the topics covered in most of the textbooks on the subject use a mixture of design, manufacture, and applications; treating only the basic physics needed to carry out practical computations [1].

However, for a variety of reasons layered media have physical relevance on their own [2, 3]. The mathematical basis for understanding their behavior is the fact that the matrix representing any lossless multilayer belongs to the group $\mathrm{SU}(1,1)$. It is known that this group is locally isomorphic to the $(2+1)$-dimensional Lorentz group $\mathrm{SO}(2,1)$ [4, 5]. This leads to a natural and complete identification between reflection and transmission coefficients of the multilayer and the parameters of the corresponding Lorentz transformation. It is, precisely, the abstract composition law of $\mathrm{SU}(1,1)$ the ultimate responsible for the curious composition law of these reflection and transmission coefficients. Moreover, this fact allows one to perform experimental tests of special relativity with simple optical measurements involving multilayers [6, 6, 8].

In this respect, another remarkable fact to be considered is that $\mathrm{SU}(1,1)$ is also isomorphic to $\mathrm{SL}(2, \mathbb{R})$, which is the natural group underlying the mathematical structure of the celebrated $A B C D$ law in first-order optics. In this paper, we also exploit this correspondence to explore intriguing connections of layered media with geometrical optics.

These purely algebraic results seem to call for a geometrical interpretation. It is difficult to overestimate the role played by geometrical ideas in all the branches of physics, particularly in special relativity. The hyperbolic geometry associated with the group SU(1,1) [or, equivalently, $\mathrm{SO}(2,1)$ ] is an established doctrine [9]. In light of these considerations, it is easy to convince oneself that this geometrical approach might provide deeper insights into the action of a multilayer in a wider unifying framework that can put forward fruitful analogies with other physical phenomena.

In consequence, it is natural to view the action of a SU(1,1) multilayer matrix as a bilinear (or Möbius) transformation on the unit disc, obtained by stereographic projection of the unit hyperboloid of $\mathrm{SO}(2,1)$. This kind of bilinear representations have been discussed in detail for the Poincaré sphere in polarization optics [10, 11], for Gaussian beam propagation [12], and are also useful in laser mode-locking and optical pulse transmission [13].

In addition, the isomorphism with $\mathrm{SL}(2, \mathbb{R})$ allows us to translate the geometrical structure defined in the unit disc to the complex plane, recovering in this way an alternative model of the hyperbolic geometry that is useful in shedding light into the behavior of the multilayer.

In spite of these achievements, the geometrical action of an arbitrary lossless multilayer could still become cumbersome to interpret in physical terms. In fact, in practice it is usual to work directly with the numerical values of a matrix obtained from the experiment, which cannot be directly related to the inner multilayer structure. To 
remedy this situation, we have resorted recently [14] to the Iwasawa decomposition, which provides a remarkable factorization of the matrix representing any multilayer (no matter how complicated it could be) as the product of three matrices of simple interpretation.

At the geometric level, such a decomposition translates directly into the classification of three basic actions, which are studied in this paper, that are the basic bricks from which any multilayer action is built.

The contents of this paper are organized as follows. In Section 2 we present in detail some relevant algebraic properties of lossless multilayers. These properties have a direct translation into hyperbolic geometry, both in the unit disc and in the complex plane, which is explored in Section 3. Finally, Section 4 devote to develop a simple yet relevant example that illustrates the power of this approach and to expose our concluding remarks.

\section{SOME PROPERTIES OF LOSSLESS MULTILAYER MATRICES}

\section{A. Transfer matrix for a lossless multilayer}

We first briefly summarize the essential ingredients of multilayer optics we shall need for our purposes 10 . The configuration is a stratified structure, illustrated in Fig. 1 , that consists of a stack of $1, \ldots, j, \ldots, m$, planeparallel lossless layers sandwiched between two semiinfinite ambient $(a)$ and substrate $(s)$ media, which we shall assume to be identical, since this is the common experimental case. Hereafter all the media are supposed to be lossless, linear, homogeneous, and isotropic.

We consider an incident monochromatic linearly polarized plane wave from the ambient, which makes an angle $\theta_{0}$ with the normal to the first interface and has amplitude $E_{a}^{(+)}$. The electric field is either in the plane of incidence ( $p$ polarization) or perpendicular to the plane of incidence ( $s$ polarization). We consider as well another plane wave of the same frequency and polarization, and with amplitude $E_{s}^{(-)}$, incident from the substrate at the same angle $\theta_{0}$. 115]

As a result of multiple reflections in all the interfaces, we have a backward-traveling plane wave in the ambient, denoted $E_{a}^{(-)}$, and a forward-traveling plane wave in the substrate, denoted $E_{s}^{(+)}$. If we consider the field amplitudes as a vector of the form

$$
\mathbf{E}=\left(\begin{array}{c}
E^{(+)} \\
E^{(-)}
\end{array}\right)
$$

which applies to both ambient and substrate media, then the amplitudes at each side of the multilayer are related by a $2 \times 2$ complex matrix $\mathrm{M}_{\text {as }}$, we shall call the multilayer transfer matrix [16], in the form

$$
\mathbf{E}_{a}=\mathrm{M}_{a s} \mathbf{E}_{s} .
$$

The matrix $\mathrm{M}_{a s}$ can be shown to be

$$
\mathbf{M}_{a s}=\left[\begin{array}{cc}
1 / T_{a s} & R_{a s}^{*} / T_{a s}^{*} \\
R_{a s} / T_{a s} & 1 / T_{a s}^{*}
\end{array}\right] \equiv\left[\begin{array}{cc}
\alpha & \beta \\
\beta^{*} & \alpha^{*}
\end{array}\right],
$$

where the complex numbers

$$
R_{a s}=\left|R_{a s}\right| \exp (i \rho), \quad T_{a s}=\left|T_{a s}\right| \exp (i \tau),
$$

are, respectively, the overall reflection and transmission coefficients for a wave incident from the ambient. Note that

$$
\operatorname{det} \mathrm{M}_{a s}=|\alpha|^{2}-|\beta|^{2}=\frac{1-\left|R_{a s}\right|^{2}}{\left|T_{a s}\right|^{2}}=+1 .
$$

Therefore, the condition $\operatorname{det} \mathrm{M}_{a s}=+1$ is equivalent to $\left|R_{a s}\right|^{2}+\left|T_{a s}\right|^{2}=1$, and then the set of lossless multilayer matrices reduces to the group $\mathrm{SU}(1,1)$, whose elements depend on three independent real parameters.

When the ambient and substrate media are different, this result also holds after a convenient renormalization of the field amplitudes 《⿶. The identity matrix corresponds to $T_{a s}=1$ and $R_{a s}=0$, so it represents an antireflection system. The matrix that describe the overall system obtained by putting two multilayers together is the product of the matrices representing each one of them, taken in the appropriate order. So, two multilayers, which are inverse, when composed give an antireflection system.

\section{B. A basic factorization for multilayers: the Iwasawa decomposition}

Many types of matrix factorizations have been considered in the literature 17, 18, 19, all of them decomposing the matrix as a unique product of other matrices of simpler interpretation. Particularly, given the essential role played by the Iwasawa decomposition, both in fundamental studies and in applications to several fields (especially in optics), one is tempted to investigate also its role in multilayer optics.

Without embarking us in mathematical subtleties, the Iwasawa decomposition is established as follows [20, 21: any element $g$ of a (noncompact semi-simple) Lie group can be written as an ordered product of three elements, taken one each from a maximal compact subgroup $K$, a maximal Abelian subgroup $A$, and a maximal nilpotent subgroup $N$. Furthermore, such a decomposition is global (in the sense that it applies to every group element) and essentially unique (in the sense that the elements of the factorization are uniquely determined in terms of $g$ ).

For the problem at hand of a lossless multilayer matrix $\mathrm{M}_{\text {as }} \in \mathrm{SU}(1,1)$, the decomposition reads as 14

$$
\mathrm{M}_{a s}=\mathrm{K}(\phi) \mathrm{A}(\xi) \mathrm{N}(\nu)
$$

where

$$
\mathrm{K}(\phi)=\left[\begin{array}{cc}
\exp (i \phi / 2) & 0 \\
0 & \exp (-i \phi / 2)
\end{array}\right]
$$




$$
\begin{aligned}
& \mathrm{A}(\xi)=\left[\begin{array}{cc}
\cosh (\xi / 2) & i \sinh (\xi / 2) \\
-i \sinh (\xi / 2) & \cosh (\xi / 2)
\end{array}\right], \\
& \mathrm{N}(\nu)=\left[\begin{array}{cc}
1-i \nu / 2 & \nu / 2 \\
\nu / 2 & 1+i \nu / 2
\end{array}\right] .
\end{aligned}
$$

The parameters $\phi, \xi$, and $\nu$ are given in terms of the elements of the multilayer matrix by

$$
\begin{aligned}
\phi / 2 & =\arg (\alpha+i \beta), \\
\xi / 2 & =\ln (1 /|\alpha+i \beta|), \\
\nu / 2 & =\operatorname{Re}\left(\alpha \beta^{*}\right) /|\alpha+i \beta|^{2},
\end{aligned}
$$

where the ranges of the parameters are $\xi, \nu \in \mathbb{R}$ and $-2 \pi \leq \phi \leq 2 \pi$. Therefore, given a priori limits on $\alpha$ and $\beta$ (i.e., on $T_{a s}$ and $R_{a s}$ ), one could easily establish the corresponding limits on the parameters $\phi, \xi$ and $\nu$, and vice versa.

All the matrices in Eq. (6) are of $\mathrm{SU}(1,1)$ and, therefore, leave invariant the expression $\left|E^{(+)}\right|^{2}-\left|E^{(-)}\right|^{2}$ at each side of the multilayer; i.e.,

$$
\left|E_{a}^{(+)}\right|^{2}-\left|E_{a}^{(-)}\right|^{2}=\left|E_{s}^{(+)}\right|^{2}-\left|E_{s}^{(-)}\right|^{2},
$$

which is nothing but the energy-flux conservation, as could be expected from physical considerations. In addition, the matrix $\mathrm{K}(\phi)$ preserves the product $E^{(+)} E^{(-)}$, the matrix $\mathrm{A}(\xi)$ preserves the quadratic form $E^{(+)^{2}}+$ $E^{(-)^{2}}$, and the matrix $\mathrm{N}(\nu)$ preserves the sum $E^{(+)}+$ $i E^{(-)}$.

\section{Connections with geometrical optics}

It this subsection we wish to show a remarkable analogy with geometrical optics that could provide new insights into the interpretation of the action of any lossless multilayer. To this end we note that by applying the unitary matrix

$$
\mathcal{U}=\frac{1}{\sqrt{2}}\left[\begin{array}{ll}
1 & i \\
i & 1
\end{array}\right]
$$

to both sides of Eq. (2), we can recast it as

$$
\mathcal{E}_{a}=\mathcal{M}_{a s} \mathcal{E}_{s}
$$

where the new field vectors are defined as

$$
\mathcal{E}=\left(\begin{array}{c}
\mathcal{E}^{(+)} \\
\mathcal{E}^{(-)}
\end{array}\right)=\mathcal{U} \mathbf{E}=\frac{1}{\sqrt{2}}\left(\begin{array}{l}
E^{(+)}+i E^{(-)} \\
E^{(-)}+i E^{(+)}
\end{array}\right),
$$

and the conjugate multilayer matrix is

$$
\mathcal{M}_{a s}=\mathcal{U} \mathrm{M}_{a s} \mathcal{U}^{-1}=\left[\begin{array}{ll}
a & b \\
c & d
\end{array}\right],
$$

where $\mathcal{M}_{a s}$ is a matrix with $\operatorname{det} \mathcal{M}_{a s}=+1$ and whose elements are real numbers given by

$$
\begin{array}{cc}
a=\operatorname{Re}(\alpha)+\operatorname{Im}(\beta), & b=\operatorname{Im}(\alpha)+\operatorname{Re}(\beta), \\
c=-\operatorname{Im}(\alpha)+\operatorname{Re}(\beta), & d=\operatorname{Re}(\alpha)-\operatorname{Im}(\beta) .
\end{array}
$$

In other words, the matrices $\mathcal{M}_{a s}$ belong to the group $\mathrm{SL}(2, \mathbb{R})$, which plays an essential role in a variety of branches in optics, ranging from the celebrated $A B C D$ law of geometrical optics [22, 23, 24, 25], to squeezed states in quantum optics [26, 27], including beam propagation problems [28]. The transformation by $\mathcal{U}$ establishes a one-to-one map between the group $\operatorname{SL}(2, \mathbb{R})$ of matrices $\mathcal{M}_{a s}$ and the group $\mathrm{SU}(1,1)$ of matrices $\mathrm{M}_{a s}$, which allows for a direct translation of the properties from one to the other. Such a correspondence is an isomorphism of groups, because

$\mathcal{M}_{a s}^{(2)} \mathcal{M}_{a s}^{(1)}=\mathcal{U} \mathrm{M}_{a s}^{(2)} \mathcal{U}^{-1} \mathcal{U} \mathrm{M}_{a s}^{(1)} \mathcal{U}^{-1}=\mathcal{U} \mathrm{M}_{a s}^{(2)} \mathrm{M}_{a s}^{(1)} \mathcal{U}^{-1}$

By conjugating with $\mathcal{U}$ the Iwasawa decomposition (6), we get the corresponding one for $\mathrm{SL}(2, \mathbb{R})$, which has been previously worked out 29]:

$$
\mathcal{M}_{a s}=\mathcal{K}(\phi) \mathcal{A}(\xi) \mathcal{N}(\nu),
$$

where

$$
\begin{aligned}
\mathcal{K}(\phi) & =\left[\begin{array}{cc}
\cos (\phi / 2) & \sin (\phi / 2) \\
-\sin (\phi / 2) & \cos (\phi / 2)
\end{array}\right], \\
\mathcal{A}(\xi) & =\left[\begin{array}{cc}
\exp (\xi / 2) & 0 \\
0 & \exp (-\xi / 2)
\end{array}\right], \\
\mathcal{N}(\nu) & =\left[\begin{array}{ll}
1 & 0 \\
\nu & 1
\end{array}\right] .
\end{aligned}
$$

Now, we can interpret the physical action of the matrices appearing in both factorizations in $\mathrm{SU}(1,1)$ and $\mathrm{SL}(2, \mathbb{R})$, respectively. In this way, $\mathrm{K}(\phi)$ represents the free propagation of the fields $\mathbf{E}$ in the ambient medium through an optical phase thickness of $\phi / 2$. Obviously, this reduces to a mere shift of the origin of phases. Alternatively, one can consider $\mathcal{K}(\phi)$ as an $A B C D$ matrix in geometrical optics that applies to position $\mathbf{x}$ and momentum $p$ (direction) coordinates of a ray in a transverse plane [30]. These are the natural phase-space variables of ray optics and then $\mathcal{K}(\phi)$ would represent a rotation in these variables [28. In the multilayer picture, $\mathcal{E}^{(+)}$can be seen as the variable $\mathbf{x}$ and $\mathcal{E}^{(-)}$can be seen as the corresponding $\mathbf{p}$.

In Eq. (7), the second matrix $A(\xi)$ represents a symmetric multilayer (i.e., the reflection coefficient is the same whether light is incident on one side or on the opposite side of the multilayer, and so $\tau_{\mathrm{A}}-\rho_{\mathrm{A}}= \pm \pi / 2$ ) with transmission and reflection phase shifts of $\tau_{\mathrm{A}}=0$ and $\rho_{\mathrm{A}}= \pm \pi / 2$, and a transmission coefficient $T_{\mathrm{A}}=$ $\operatorname{sech}(\xi / 2)$. There are many ways to get this performance, perhaps the simplest one is a Fabry-Perot system composed by two identical plates separated by a transparent spacer. By adjusting the refractive indices and the thicknesses of the media one can always get the desired values. Viewed in $\operatorname{SL}(2, \mathbb{R}), \mathcal{A}(\xi)$ represents a magnifier that scales $\mathbf{x}$ up to the factor $m=\exp (\xi / 2)$ and $\mathbf{p}$ down by the same factor 28 .

The third matrix, $\mathrm{N}(\nu)$, represents a system having $T_{\mathrm{N}}=\cos \left(\tau_{\mathrm{N}}\right) \exp \left(i \tau_{\mathrm{N}}\right)$ and $R_{\mathrm{N}}=\sin \left(\tau_{\mathrm{N}}\right) \exp \left(i \tau_{\mathrm{N}}\right)$, with 
$\tan \left(\tau_{\mathrm{N}}\right)=\nu / 2$. The simplest way to accomplish this task is by an asymmetrical two-layer system. Using the analogy with the $A B C D$ matrix in geometrical optics, $\mathcal{N}(\nu)$ represents the action of a lens of power $\nu$ 28.

Finally, in complete equivalence with the invariants found for the Iwasawa decomposition of SU(1,1), the matrix $\mathcal{K}$ preserves the sum $\mathcal{E}^{(+)^{2}}+\mathcal{E}^{(-)^{2}}, \mathcal{A}$ preserves the product $\mathcal{E}^{(+)} \mathcal{E}^{(-)}$, and $\mathcal{N}$ preserves $\mathcal{E}^{(+)}$. In addition, the energy-flux conservation Eq. (9) can be recast now as the invariance of $\operatorname{Im}\left[\mathcal{E}^{(+)} \mathcal{E}^{(-)^{*}}\right]$.

\section{Connections with special relativity}

Apart from the geometrical-optics perspective developed in the previous subsection, multilayer action can be viewed in a relativisticlike framework, that has proved to be very appropriate to understand some peculiarities of multilayer behavior. To this end, let us first recall some well-known facts about the Lorentz transformations in $(2+1)$-dimensions.

Introducing a three-dimensional real vector space of vectors with components $\left(x^{0}, x^{1}, x^{2}\right)$, where $x^{0}=c t$, a Lorentz transformation $\Lambda$ is a linear transformation between two coordinate frames

$$
x^{\prime \mu}=\Lambda_{\nu}^{\mu} x^{\nu}
$$

(the Greek indices run from 0 to 2), such that the pseudoEuclidean bilinear form

$$
\langle x \mid y\rangle=x^{0} y^{0}-x^{1} y^{1}-x^{2} y^{2}
$$

remains invariant; i.e., $\left\langle x^{\prime} \mid y^{\prime}\right\rangle=\langle x \mid y\rangle$, which immediately implies

$$
\operatorname{det} \Lambda= \pm 1
$$

Thus, the set of Lorentz transformations can be classified in two classes: proper transformations, with $\operatorname{det} \Lambda=+1$, and improper ones, with $\operatorname{det} \Lambda=-1$. The proper Lorentz transformations form a subgroup, but the improper ones do not.

Furthermore, it is easy to check that $\left|\Lambda^{0}{ }_{0}\right| \geq 1$. Therefore, the transformations of the Lorentz group can also be classified according to the sign of $\Lambda^{0}{ }_{0}$ : the orthochronous Lorentz transformations, with $\Lambda_{0}^{0} \geq 1$, form a subgroup, but the antichronous ones, with $\Lambda^{0}{ }_{0} \leq-1$, do not.

We are interested in dealing with the three-parameter restricted Lorentz group $\mathrm{SO}(2,1)$; i.e., the group of the Lorentz transformations with determinant +1 and that do not reverse the direction of time. In fact, we wish to discuss now a very close correspondence between the group $\mathrm{SU}(1,1)$ introduced above and the restricted Lorentz group $\mathrm{SO}(2,1)$. We shall show this important correspondence explicitly in the following form that we recall for clarity 31]: with each point of our threedimensional vector space with coordinates $x^{\mu}$ we associate the Hermitian matrix

$$
\mathrm{X}=x^{\mu} \sigma_{\mu}=\left[\begin{array}{cc}
x^{0} & x^{1}-i x^{2} \\
x^{1}+i x^{2} & x^{0}
\end{array}\right]
$$

where $\sigma_{0}=I$ is the identity, and $\sigma_{1}$ and $\sigma_{2}$ are the corresponding Pauli matrices. Note, that $\operatorname{det} \mathrm{X}=\langle x \mid x\rangle=$ $\left(x^{0}\right)^{2}-\left(x^{1}\right)^{2}-\left(x^{2}\right)^{2}$.

Now, if $\mathrm{M} \in \mathrm{SU}(1,1)$, then the matrix

$$
\mathrm{X}^{\prime}=\mathrm{M} \times \mathrm{M}^{\dagger}
$$

where the symbol $\dagger$ denotes the Hermitian conjugate, induces a Lorentz transformation on the coefficients $x^{\mu}$.

It is clear from Eq. (22) that the matrices $M$ and $-M$ generate the same $\Lambda$, so this homomorphism is two-toone. This equation can be easily solved to obtain $M$ (uniquely defined up to the sign) from a given $\Lambda$. In fact, it is easy to find it explicitly as [20]

$$
\Lambda_{\nu}^{\mu}(\mathrm{M})=\frac{1}{2} \operatorname{Tr}\left(\sigma^{\mu} \mathrm{M} \sigma_{\nu} \mathrm{M}^{\dagger}\right) .
$$

While $\mathrm{M}$ acts on two-dimensional complex vectors like those in Eq. (1), the induced Lorentz transformation $\Lambda(\mathrm{M})$ acts on three-dimensional real vectors of the form [which are the space-time counterparts of Eq. (1)]:

$$
\left(\begin{array}{c}
x^{0} \\
x^{1} \\
x^{2}
\end{array}\right) \rightarrow\left(\begin{array}{c}
e^{0} \\
e^{1} \\
e^{2}
\end{array}\right)=\left(\begin{array}{c}
\left(\left|E^{(+)}\right|^{2}+\left|E^{(-)}\right|^{2}\right) / 2 \\
\operatorname{Re}\left[E^{(+)^{*}} E^{(-)}\right] \\
\operatorname{Im}\left[E^{(+)^{*}} E^{(-)}\right]
\end{array}\right)
$$

The temporal coordinate is the semi-sum of the fluxes at each side of the multilayer. The interval remains invariant

$$
\left(e^{0}\right)^{2}-\left(e^{1}\right)^{2}-\left(e^{2}\right)^{2}=K^{2} .
$$

This number $K$ (which is the 'radius') is the semidifference of the fluxes at each side of the multilayer; i.e., $\left(\left|E^{(+)}\right|^{2}-\left|E^{(-)}\right|^{2}\right) / 4$ for both ambient or substrate and, therefore, it can take any real value. Without loss of generality we can renormalize these variables so as to take the value of $K$ equal to 1 and then we are working on the unit two-sheeted hyperboloid of $\operatorname{SO}(2,1)$ [32].

In summary, given the multilayer matrix $\vec{M}_{a s}$ in Eq. (3), the corresponding Lorentz transformation in $\mathrm{SO}(2,1)$ is

$$
\Lambda\left(\mathrm{M}_{a s}\right)=\left[\begin{array}{ccc}
|\alpha|^{2}+|\beta|^{2} & 2 \operatorname{Re}\left(\alpha \beta^{*}\right) & 2 \operatorname{Im}\left(\alpha \beta^{*}\right) \\
2 \operatorname{Re}(\alpha \beta) & \operatorname{Re}\left(\alpha^{2}+\beta^{2}\right) & \operatorname{Im}\left(\alpha^{2}-\beta^{2}\right) \\
-2 \operatorname{Im}(\alpha \beta) & -\operatorname{Im}\left(\alpha^{2}+\beta^{2}\right) & \operatorname{Re}\left(\alpha^{2}-\beta^{2}\right)
\end{array}\right] .
$$

Moreover, using Eq. (23) it is direct to obtain the explicit expressions in $\mathrm{SO}(2,1)$ for each matrix appearing in the Iwasawa decomposition (6). Indeed one finds

$$
\begin{aligned}
\Lambda_{\mathrm{K}}(\phi)= & {\left[\begin{array}{ccc}
1 & 0 & 0 \\
0 & \cos \phi & \sin \phi \\
0 & -\sin \phi & \cos \phi
\end{array}\right], } \\
\Lambda_{\mathrm{A}}(\xi)= & {\left[\begin{array}{ccc}
\cosh \xi & 0 & -\sinh \xi \\
0 & 1 & 0 \\
-\sinh \xi & 0 & \cosh \xi
\end{array}\right], } \\
\Lambda_{\mathrm{N}}(\nu)= & {\left[\begin{array}{ccc}
1+\left(\nu^{2} / 2\right) & \nu & -\nu^{2} / 2 \\
\nu & 1 & -\nu \\
\nu^{2} / 2 & \nu & 1-\left(\nu^{2} / 2\right)
\end{array}\right] . }
\end{aligned}
$$


The action of these matrices in $\mathrm{SO}(2,1)$ is clear: $\Lambda_{\mathrm{K}}(\phi)$ is a space rotation of angle $\phi$ in the $e^{1}-e^{2}$ plane, $\Lambda_{\mathrm{A}}(\xi)$ is a boost in the direction of the axis $e^{2}$ with velocity $v / c=$ $\tanh \xi$; and, finally, $\Lambda_{\mathrm{N}}(\nu)$ represents a space rotation of angle $\tau_{\mathrm{N}}$ [such that $\tan \left(\tau_{\mathrm{N}}\right)=\nu / 2$ ] followed by a boost of angle $\tau_{\mathrm{N}}$ and velocity $v / c=\tanh (\nu / 2)$, both in the $e^{1}-e^{2}$ plane. In the next Section we shall explore in more detail the properties of these three matrices.

\section{GEOMETRICAL INTERPRETATION OF THE MULTILAYER ACTION}

\section{A. Multilayer transfer function and hyperbolic geometry in the unit disc}

In many instances (e.g., in polarization optics [10]) we are interested in the transformation properties of field quotients rather than the fields themselves. Therefore, it seems natural to consider the complex numbers

$$
z_{s}=\frac{E_{s}^{(-)}}{E_{s}^{(+)}}, \quad z_{a}=\frac{E_{a}^{(-)}}{E_{a}^{(+)}} .
$$

The action of the multilayer given in Eq. (3) can be then seen as a function $z_{a}=f\left(z_{s}\right)$ that can be appropriately called the multilayer transfer function [16.

From a geometrical viewpoint, this function defines a transformation of the complex plane $\mathbb{C}$, mapping the point $z_{s}$ into the point $z_{a}$, according to

$$
z_{a}=\Phi\left[\mathrm{M}_{a s}, z_{s}\right]=\frac{\beta^{*}+\alpha^{*} z_{s}}{\alpha+\beta z_{s}},
$$

and the point of the infinity is given by

$$
\Phi\left[\mathrm{M}_{a s},-\alpha / \beta\right]=\infty, \quad \Phi\left[\mathrm{M}_{a s}, \infty\right]=\alpha^{*} / \beta .
$$

This bilinear transformation defines an action of the group SU(1,1) of multilayer transfer matrices on the complex plane $\mathbb{C}$. The complex plane appears then foliated in three regions that remain invariant under the action of the group: the unit disc, its boundary and the external region. In fact,

$$
\left|z_{a}\right|^{2}=\frac{|\alpha|^{2}\left|z_{s}\right|^{2}+|\beta|^{2}+2 \operatorname{Re}\left(\beta \alpha^{*} z_{s}\right)}{|\alpha|^{2}+|\beta|^{2}\left|z_{s}\right|^{2}+2 \operatorname{Re}\left(\beta \alpha^{*} z_{s}\right)},
$$

and, in consequence, the difference between the numerator and the denominator is

$$
\left(|\alpha|^{2}-|\beta|^{2}\right)\left(\left|z_{s}\right|^{2}-1\right)=\left|z_{s}\right|^{2}-1
$$

which shows our assertion relative to the invariance of those regions.

Alternatively, the unit disc can be seen as obtained from the upper sheet of the unit two-sheet hyperboloid defined by Eq. (25) with $K=1$ by means of stereographic projection using the south pole $(-1,0,0)$ as projection centre. In fact, a simple calculation shows that, in such a case, the projection of the point $\left(e^{0}, e^{1}, e^{2}\right)$ becomes in the complex plane

$$
z=\frac{e^{1}+i e^{2}}{1+e^{0}}=\frac{E^{(-)}}{E^{(+)}},
$$

in full agreement with Eq. (28).

The boundary of the unit disc corresponds to the projection of the infinity point and then, since $\left|z_{a}\right|=\left|z_{s}\right|=$ 1 , it can be identified with the action of perfect mirrors (i.e., $\left.T_{a s}=0\right)$.

The Iwasawa decomposition has an immediate translation in this geometrical framework, and one is led to treat separately the action of each one of the matrices appearing in this decomposition. To this end, it is worth noting that the groups we are considering appear always as groups of transformations of some space. The concept of orbit is especially appropriate for obtaining an intuitive meaning of the corresponding action. We recall that, given a point $P$, its orbit is the set of points $P^{\prime}$ obtained from $P$ by the action of all the elements of the group. In Fig. 2 we have plotted a typical example of the orbits for each one of the subgroups of matrices $\Lambda_{\mathrm{K}}(\phi)$, $\Lambda_{\mathrm{A}}(\xi)$, and $\Lambda_{\mathrm{N}}(\nu)$. For $\Lambda_{\mathrm{K}}(\phi)$ the orbits are the intersection of the hyperboloid with planes $e^{0}=$ constant, for $\Lambda_{\mathrm{A}}(\xi)$ with planes $e^{1}=$ constant, and for $\Lambda_{\mathrm{N}}(\nu)$ with planes $e^{0}-e^{2}=$ constant.

Through stereographic projection, as indicated in Fig. 2, we are working in the unit disc and the corresponding orbits for the $\mathrm{SU}(1,1)$ matrices $\mathrm{K}, \mathrm{A}$, and $\mathrm{N}$ are

$$
\begin{aligned}
& z^{\prime}=\Phi[\mathrm{K}(\phi), z]=z \exp (-i \phi), \\
& z^{\prime}=\Phi[\mathrm{A}(\xi), z]=\frac{z-i \tanh (\xi / 2)}{1+i z \tanh (\xi / 2)}, \\
& z^{\prime}=\Phi[\mathrm{N}(\nu), z]=\frac{z+(1+i z) \nu / 2}{1+(z-i) \nu / 2} .
\end{aligned}
$$

As plotted in Fig. 3.a, for matrices $\mathrm{K}$ the orbits are circumferences centered at the origin. Since for these matrices $R_{a s}=0$, the action of any antireflection system can be always pictured as one of these circumferences. For the matrices $A$, they are arcs of circumference centered in the real axis and going from the point $+i$ to the point $-i$ through $z$. Finally, for the matrices $\mathrm{N}$ the orbits are circumferences centered in the imaginary axis and passing all of them through the points $i, z$, and $-z^{*}$.

The importance of the Iwasawa decomposition reflects also at the geometrical level: no matter how complicated a multilayer is, its action can always be viewed in terms of these three basic actions with a clear geometric meaning. Its explicit application to a real case will be demonstrated in the next Section.

\section{B. Hyperbolic geometry in the upper semi-plane}

The unitary transformation (10) has played an important role as intertwining between multilayer and geometrical optics. One can expect that the structure defined in 
the unit disc for the former could be translated accordingly for the latter. To this end, note that if the point $w \in \mathbb{C}$ is defined in terms of $z$ by

$$
w=\Phi[\mathcal{U}, z]=\frac{z+i}{1+i z},
$$

it is easy to check that the interior of the unit disc is mapped onto the upper semi-plane of the complex plane $w$, the boundary maps onto the real axis, while the exterior of the unit disc becomes the lower semi-plane.

The relationship between $\mathrm{SU}(1,1)$ and $\mathrm{SL}(2, \mathbb{R})$ outlined in Section 2 allows us to transport the action of $\mathrm{SU}(1,1)$ onto $\mathbb{C}$ to give an action $\Psi$ of $\mathrm{SL}(2, \mathbb{R})$ onto $\mathbb{C}$, finding in this way an alternative model of the hyperbolic geometry and for determining the orbits of the matrices of $\mathrm{SL}(2, \mathbb{R})$, that are the natural arena of geometrical optics.

The corresponding orbits are now

$$
\begin{aligned}
w^{\prime} & =\Psi[\mathcal{K}(\phi), w]=\frac{w-\tan (\phi / 2)}{1+w \tan (\phi / 2)}, \\
w^{\prime} & =\Psi[\mathcal{A}(\xi), w]=w \exp (-\xi), \\
w^{\prime} & =\Psi[\mathcal{N}(\nu), w]=w+\nu .
\end{aligned}
$$

For matrices $\mathcal{K}$ the orbits are circumferences centered in the imaginary axis passing through $w$ and $-1 / w$. For the matrices $\mathcal{A}$, they are straight lines in the upper semiplane passing through the origin and the point $w$. Finally, for the matrices $\mathcal{N}$, we have straight lines parallel to the real axis passing through the point $w$. In Fig. 3.b we have plotted these orbits in the complex plane $w$.

\section{A SIMPLE EXAMPLE AND CONCLUDING REMARKS}

It seems pertinent to conclude by showing the power of this geometrical approach. In consequence, we shall analyze in some detail a practical example: a single glass plate of refractive index $n_{1}=1.5$ and thickness $d_{1}=1.1$ $\mathrm{mm}$ embedded in air. The plate is illuminated with a monochromatic light of wavelength in vacuo $\lambda=546 \mathrm{~nm}$ that impinges from both ambient and substrate at an angle $\theta_{0}=45^{\circ}$.

For this system a standard calculation gives the following reflection and transmission coefficients:

$$
R_{a s}=\frac{r_{01}\left[1-\exp \left(-i 2 \beta_{1}\right)\right]}{1-r_{01}^{2} \exp \left(-i 2 \beta_{1}\right)}
$$

$$
T_{a s}=\frac{\left(1-r_{01}^{2}\right) \exp \left(-i \beta_{1}\right)}{1-r_{01}^{2} \exp \left(-i 2 \beta_{1}\right)}
$$

where $r_{01}$ is the Fresnel reflection coefficient at the interface 01 (which applies to both $p$ and $s$ polarizations by the simple attachment of a subscript $p$ or $s$ ) and $\beta_{1}$ is the plate phase thickness

$$
\beta_{1}=\frac{2 \pi}{\lambda} n_{1} d_{1} \cos \theta_{1}
$$

If we take as initial condition that in the substrate $z_{s}=0.5 \exp (i \pi / 6)$, then we obtain from Eqs. (37) the value $z_{a}=-0.6149+0.0498 i$ (for $s$ polarization). In Fig. 4 we have plotted these points $z_{s}$ and $z_{a}$ in the unit disc. Obviously, from these (experimental) data alone we cannot infer at all the possible path for this discrete transformation.

However, the Iwasawa decomposition remedies this serious drawback: from the geometrical meaning discussed before, and once we know the values of $\phi, \xi$, and $\nu$ [that are easily computed from Eqs. (8)] we get the intermediate values of $z^{\prime}$ for the ordered application of the matrices $\mathrm{K}(\phi), \mathbf{A}(\xi)$, and $\mathbf{N}(\nu)$, which, in fact, determines that the trajectory from $z_{s}$ to $z_{a}$ is well defined through the corresponding orbits, as shown in Fig. 4.

Moreover, and this is the important moral we wish to extract from this simple example, if in some experiment the values of $z_{s}$ and $z_{a}$ are measured, one can find, no matter how complicated the multilayer is, in a unique way, the three arcs of orbits that connect the initial and final points in the unit disc.

We stress that the benefit of this approach lies not in any inherent advantage in terms of efficiency in solving problems in layered structures. Rather, we expect that the formalism presented here could provide a general and unifying tool to analyze multilayer performance in an elegant and concise way that, additionally, is closely related to other fields of physics, such as special relativity and geometrical optics.

\section{Acknowledgments}

We wish to thank J. Zoido and C. Criado for their help in computing some of the figures of this paper.
[1] H. A. Macleod, Thin-film Optical Filters (Adam Hilger, Bristol, 1986).

[2] P. Yeh, Optical Waves in Layered Media (Wiley, New York, 1988).

[3] J. Lekner, Theory of Reflection (Dordrecht, Amsterdam, 1987).
[4] J. J. Monzón and L. L. Sánchez-Soto, "Lossles multilayers and Lorentz transformations: more than an analogy," Opt. Commun. 162, 1-6 (1999).

[5] J. J. Monzón and L. L. Sánchez-Soto, "Fully relativisticlike formulation of multilayer optics," J. Opt. Soc. Am. A 16, 2013-2018 (1999). 
[6] J. J. Monzón and L. L. Sánchez-Soto, "Origin of the Thomas rotation that arises in lossless multilayers," J. Opt. Soc. Am. A 16, 2786-2792 (1999).

[7] J. J. Monzón and L. L. Sánchez-Soto, "Multilayer optics as an analog computer for testing special realtivity," Phys. Lett. A 262, 18-26 (1999).

[8] J. J. Monzón and L. L. Sánchez-Soto, "A simple optical demostration of geometric phases from multilayer stacks: the Wigner angle as an anholonomy," J. Mod. Opt. 48, 21-34 (2001).

[9] H. S. M. Coxeter, Non-Euclidean Geometry (University of Toronto Press, Toronto, 1968).

[10] R. M. A. Azzam and N. M. Bashara, Ellipsometry and Polarized Light (North-Holland, Amsterdam, 1987).

[11] D. Han, Y. S. Kim, and M. E. Noz, "Polarization optics and bilinear representations of the Lorentz group," Phys. Lett. A 219, 26-32 (1996).

[12] H. Kogelnik, "Imaging of optical modes -resonators with internal lenses," Bell Syst. Techn. J. 44, 455-494 (1965).

[13] M. Nakazawa, J. H. Kubota, A. Sahara, and K. Tamura, "Time-domain ABCD matrix formalism for laser modelocking and optical pulse transmission," IEEE J. Quant. Electron. QE34, 1075-1081 (1998).

[14] J. J. Monzón, T. Yonte, and L. L. Sánchez-Soto, "Basic factorization for multilayers," Opt. Lett. 26, 370-372 (2001).

[15] When ambient $(0)$ and substrate $(m+1)$ media are different, the angles $\theta_{0}$ and $\theta_{m+1}$ are conected by Snell law $n_{0} \sin \theta_{0}=n_{m+1} \sin \theta_{m+1}$, where $n_{j}$ denotes the refractive index of the $j$-th medium.

[16] I. Ohlídal and D. Franta, Ellipsometry of Thin Film Systems, Progress in Optics (Edited by E. Wolf) 41, 181 (North-Holland, Amsterdam, 2000).

[17] H. H. Arsenault and B. Macukow, "Factorization of the transfer matrix for symmetrical optical systems," J. Opt. Soc. Am. 73, 1350-1359 (1983).

[18] S. Abe and J. T. Sheridan, "Optical operations on wave functions as the Abelian subgroups of the special affine Fourier transformation," Opt. Lett. 19, 1801-1803 (1994).

[19] J. Shamir and N. Cohen, "Root and power transformations in optics," J. Opt. Soc. Am. A 12, 2415-2423 (1995).

[20] A. O. Barut and R. Rączka, Theory of Group Representations and Applications (PWN, Warszaw, 1977).

[21] S. Helgason, Differential Geometry, Lie Groups and Symmetric Spaces (Academic, New York, 1978).

[22] H. Bacry and M. Cadilhac, "The metaplectic group and Fourier optics," Phys. Rev. A 23, 2533-2536 (1981).

[23] M. Nazarathy and J. Shamir, "First order systems -a canonical operator representation: lossless systems," J. Opt. Soc. Am. 72, 356-364 (1982).

[24] E. C. G. Sudarshan, N. Mukunda, and R. Simon, "Realization of first order optical systems using thin lenses," Opt. Acta 32, 855-872 (1985).

[25] R. Simon, N. Mukunda, and E. C. G. Sudarshan, "Partially coherent beams and a generalized abcd-law," Opt. Commun. 65, 322-328 (1988).

[26] R. Simon and N. Mukunda, "Bargmann invariant and the geometry of the Gouy effect," Phys. Rev. Lett. 70, 880-883 (1993).

[27] G. S. Agarwal and R. Simon, "An experiment for the study of the Gouy effect for the squeezed vacuum," Opt. Commun. 100, 411-414 (1993).

[28] R. Simon and N. Mukunda, "Iwasawa decomposition in first-order optics: universal treatment of shape-invariant propagation for coherent and partially coherent beams," J. Opt. Soc. Am. A 15, 2146-2155 (1998).

[29] R. Simon, E. C. G. Sudarshan, and N. Mukunda, "Generalized rays in first order optics: transformation properties of Gaussian Schell-model fields," Phys. Rev. A 29, 3273-3279 (1984).

[30] J.F. Cariñena and J. Nasarre, "On symplectic structures arising from geometric optics," Forts. Phys. 44, 181-198 (1996).

[31] V. Bargmann, "Irreducible unitary representations of the Lorentz group," Ann. Math. 48, 568-640 (1947).

[32] A. Mischenko and A. Fomenko, A Course of Differential Geometry and Topology (MIR, Moscow, 1988), Sec. 1.4. 
FIG. 1: Wave vectors of the input $\left[E_{a}^{(+)}\right.$and $\left.E_{s}^{(-)}\right]$and output $\left[E_{a}^{(-)}\right.$and $\left.E_{s}^{(+)}\right]$fields in a multilayer sandwiched between two identical semi-infinite ambient and substrate media.

FIG. 2: Unit hyperboloids defined in Eq. (25), which represent the space of field states for $\mathrm{SO}(2,1)$. In each one of them we have plotted a typical orbit for the matrices: a) $\Lambda_{K}$, b) $\Lambda_{\mathrm{A}}$, and c) $\Lambda_{N}$. In all the figures we have performed stereographic projection from the south pole $S$ of the hyperboloid, to obtain the unit disc in the plane $e^{0}=0$ and the corresponding orbits, which represent the actions of the $\mathrm{SU}(1,1)$ matrices: a) $\mathrm{K}, \mathrm{b}$ ) $\mathrm{A}$, and c) $\mathrm{N}$.
FIG. 3: a) Plot of several orbits in the unit disc of the elements of the Iwasawa decomposition K, A, and N for SU(1,1) (from left to right, respectively). b) Corresponding orbits in the upper complex semiplane for the Iwasawa decomposition $\mathcal{K}$, $\mathcal{A}$, and $\mathcal{N}$ for $\mathrm{SL}(2, \mathbb{R})$. 
FIG. 4: Geometrical representation in the unit disc of the action of a single glass plate with the parameters indicated in the text. The point $z_{s}$ is transformed by the plate into the point $z_{a}$. We indicate the three orbits given by the Iwasawa decomposition and, as a thick line, the trajectory associated to the plate action. 


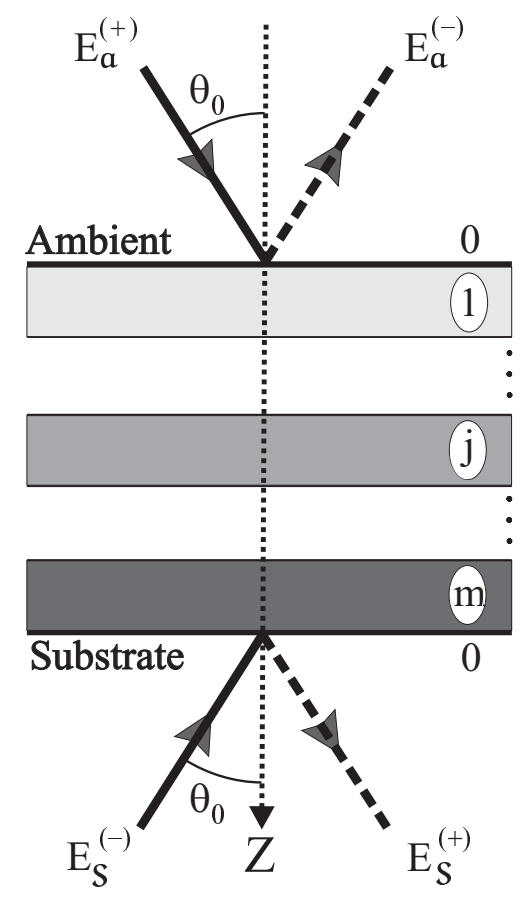


This figure "figure2.gif" is available in "gif" format from: http://arxiv.org/ps/physics/0104050v1 

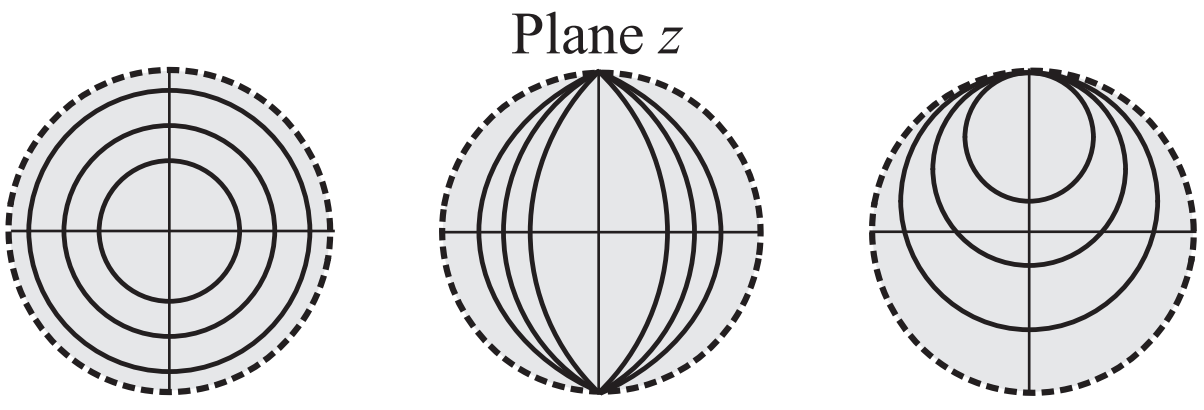

(a)

Plane $w$
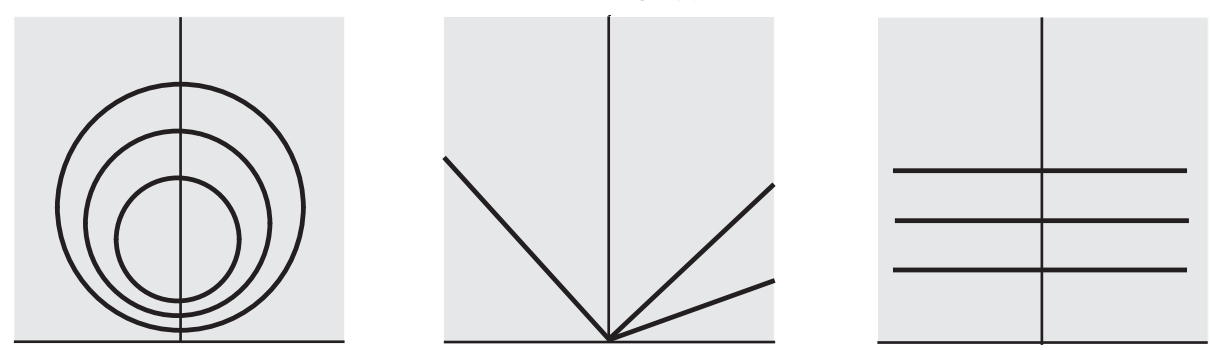

(b) 


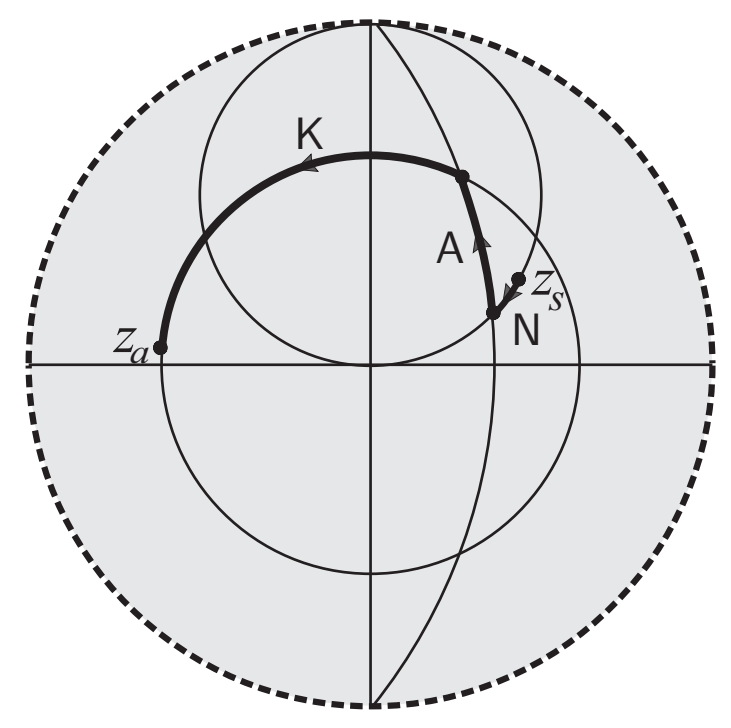

